\title{
Prevenção do infarto agudo do miocárdio feita pelos enfermeiros em uma clínica da
}

\section{família de Realengo, Rio de Janeiro}

\author{
Prevention of acute myocardial infarction by nurses at a family clinic in Realengo, Rio de Janeiro \\ Prevención del infarto agudo de miocardio por enfermeras en una clínica de la familia en Realengo,
}

Rio de Janeiro

Recebido: 03/02/2022 | Revisado: 07/02/2022 | Aceito: 13/02/2022 | Publicado: 20/02/2022

\author{
Arthur Novaes Cidade \\ ORCID: https://orcid.org/0000-0001-5267-2915 \\ Universidade Castelo Branco, Brasil \\ E-mail: arthur888.novaes@gmail.com \\ Amanda Gabriel Pimentel \\ ORCID: https://orcid.org/0000-0002-4557-3801 \\ Universidade Castelo Branco, Brasil \\ E-mail: amandagabriel1998@gmail.com \\ Maria Regina Bernardo da Silva \\ ORCID: https://orcid.org/0000-0002-3620-3091 \\ Centro Universitário CBM, Brasil \\ E-mail: m.regina2000@uol.com.br \\ Thaís Araujo Vianna \\ ORCID: https://orcid.org/0000-0002-0892-5898 \\ Universidade Castelo Branco, Brasil \\ E-mail: thais.pnk@gmail.com \\ Sandra Conceição Ribeiro Chícharo \\ ORCID: https://orcid.org/0000-0002-1487-0088 \\ Universidade Castelo Branco, Brasil \\ E-mail: sandracrchicharo@gmail.com \\ Lígia D’arc Silva Rocha Prado \\ ORCID: https://orcid.org/0000-0001-9690-9953 \\ Universidade Castelo Branco, Brasil \\ E-mail: enfaligiaprado@ hotmail.com
}

\begin{abstract}
Resumo
Objetivo: Identificar a prevenção do infarto agudo do miocárdio por intermédio dos enfermeiros de uma clínica da família na zona oeste do Rio de Janeiro. Metodologia: Pesquisa tipo qualitativa de campo realizada em uma unidade Básica com enfermeiros sendo um preceptor e oito residentes de enfermagem, $\mathrm{N}^{\circ}$ do Parecer: 4.876.209. Resultados Relataram a baixa frequência de consultas durante a pandemia da Covid-19, Observou-se a autonomia que o enfermeiro possui em relação ao acompanhamento e prescrição de acordo com os programas do ministério da saúde, Foi visto que durante todo o período da pandemia, o atendimento de usuários crônicos, estava sendo feito por demanda espontânea através de renovação de receitas e teve uma redução de atendimento e atividades de educação permanente, por conta da contingência e restrições sanitárias na unidade de saúde, devido a COVID 19, Acontecia também algumas práticas não farmacológicas aos usuários em relação a alimentação e cuidados. Conclusão: A atividade de educação permanente acontece nesta clínica da família, e os enfermeiros são envolvidos nos programas de saúde e atividades afins de educação, mas neste período de Pandemia pelo acúmulo de atendimentos, adoecimento dos profissionais, ocorrência da imunização e exames para detectar COVID 19, não estava acontecendo atividades de educação permanente.
\end{abstract}

Palavra-chave: Infarto agudo do miocárdio; Prevenção primária; Enfermagem; Autocuidado.

\begin{abstract}
Objective: To identify the prevention of acute myocardial infarction through the nurses of a family clinic in the west of Rio de Janeiro. Methodology: Qualitative field research carried out in a Basic unit with nurses, one preceptor and eight nursing residents, Opinion No.: 4,876,209. Results They reported the low frequency of consultations during the Covid-19 pandemic, It was observed the autonomy that the nurse has in relation to monitoring and prescription according to the programs of the Ministry of Health, It was seen that throughout the period of the pandemic, the care of chronic users, was being done by spontaneous demand through the renewal of recipes and there was a reduction in care and permanent education activities, due to the contingency and sanitary restrictions in the health unit, due to COVID 19, there were also some practices non-pharmacological to users in relation to food and care. Conclusion: The
\end{abstract}


continuing education activity takes place in this family clinic, and nurses are involved in health programs and related education activities, but in this period of Pandemic due to the accumulation of calls, illness of professionals, occurrence of immunization and exams to detect COVID 19, there were no continuing education activities.

Keywords: Acute myocardial infarction; Primary prevention; Nursing; Self care.

\section{Resumen}

Objetivo: Identificar la prevención del infarto agudo de miocardio a través de los enfermeros de una clínica de la familia en el oeste de Río de Janeiro. Metodología: Investigación cualitativa de campo realizada en una Unidad Básica con enfermeras, una preceptora y ocho residentes de enfermería, Dictamen $\mathrm{N}^{\circ}: 4.876 .209$. Resultados Refirieron la baja frecuencia de consultas durante la pandemia de Covid-19, Se observó la autonomía que tiene el enfermero en relación al seguimiento y prescripción de acuerdo a los programas del Ministerio de Salud, Se vio que durante todo el periodo de la pandemia , la atención de usuarios crónicos, se estaba haciendo por demanda espontánea a través de la renovación de recetas y hubo una reducción en las actividades de atención y educación permanente, debido a la contingencia y restricciones sanitarias en la unidad de salud, por el COVID 19, también hubo algunas prácticas no farmacológicas a los usuarios en relación con la alimentación y el cuidado. Conclusión: La actividad de educación continua se da en esta clínica familiar, y los enfermeros están involucrados en los programas de salud y actividades de educación afines, pero en este período de Pandemia debido a la acumulación de llamadas, enfermedad de los profesionales, ocurrencia de inmunizaciones y exámenes para detección de COVID 19, no hubo actividades de educación continua.

Palabras clave: Infarto agudo de miocardio; Prevención primaria; Enfermería; Cuidados personales.

\section{Introdução}

O infarto agudo do miocárdio (IAM), é uma síndrome de elevada prevalência no Brasil e no mundo, devido o envelhecimento populacional, baixos níveis de controle da pressão arterial (PA) e os alimentos ricos em sódio e gorduras que são bem comuns em nosso dia a dia (Andrade,2015).

As doenças cardiovasculares, incluindo o infarto agudo do miocárdio (IAM), representam um importante problema de saúde pública no Brasil e no mundo, apresentando altas taxas de incidência e mortalidade (Santos, 2021).

A Atenção Primária à Saúde (APS) é o primeiro nível de atenção e caracteriza-se por um conjunto de ações no âmbito individual e coletivo, que abrangem a promoção e a proteção da saúde, a prevenção de agravos, o diagnóstico, o tratamento, a reabilitação e a manutenção da saúde. Destaca-se que o Ministério da Saúde (MS) na Portaria que aprova a Política Nacional de Atenção Básica (PNAB) considera equivalentes os termos "Atenção Básica (AB)", "Estratégia de Saúde da Família (ESF)" e "Atenção Primária à Saúde (APS)", utilizados no Brasil. Neste artigo optou-se por utilizar a terminologia APS (Arquivos brasileiros de cardiologia, 2015).

Com a expansão e estruturação da oferta de serviços, durante o processo de construção do Sistema Único de Saúde (SUS) brasileiro, em que os municípios têm assumido a responsabilidade pela atenção à saúde de seus munícipes, sobretudo, da rede de Atenção Básica, o debate sobre o acesso a essas ações e serviços ganhou nuances qualitativas (Souza,2015).

Pesquisa realizada pelas universidades federais do Rio de Janeiro (UFRJ) e Minas Gerais (UFMG), pela Sociedade Brasileira de Cardiologia (SBC) mostram que em 2020 tem em incidência de óbitos por infarto e problemas cardiovasculares um aumento de 38,7\% no Rio de Janeiro durante a pandemia da Covid-19. Sendo comparados de 17 de março a 22 de maio deste ano com o mesmo período de 2019 (Prieto,2021).

Os fatores de risco para o desenvolvimento do infarto podem ser classificados como modificáveis e não modificáveis, os modificáveis são baseados no estilo de vida que incluem a alimentação desregrada, rica em carboidratos, gorduras, sódio e alimentos processados, o uso do álcool, do cigarro e de outras drogas e situações recorrentes de estresse e o sedentarismo, já os não modificáveis mais comuns são: a raça, a idade, o sexo, e o histórico familiar (Brunori,2015). 


\section{Metodologia}

A metodologia do projeto de conclusão de curso trata-se de um estudo de campo do tipo exploratório descritivo com abordagem qualitativa. É descrito como método qualitativo aquele que pode ser definido e aplicado ao estudo da história, das relações, das representações, das crenças, das percepções e das opiniões, produtos das interpretações que os humanos fazem a respeito de como vivem, constroem seus artefactos e a si mesmos, sentem e pensam. A pesquisa qualitativa tem como base informações exteriores nas quais serão melhoradas com o passar dos anos e que nas quais serão utilizadas para influenciar uma comunidade (Bolzan, 2020).

Cientificamente uma pesquisa qualitativa é o revisar de uma literatura sobre a mesma, o que chama atenção imediata é o fato de que, frequentemente, a pesquisa qualitativa não é sendo definida por si só, ela é um conjunto de caracterizadas que são: características da pesquisa qualitativa; postura do pesquisador; estratégias de coleta de dados; estudo de caso; papel do sujeito e aplicabilidade e uso dos resultados da pesquisa (Atlas, 2021).

A pesquisa foi realizada na Clínica da Família, situada no bairro de Realengo, na zona Oeste do Município do Rio de Janeiro, autorizada pelo comitê de ética com número de parecer: 4.876.209. O bairro em questão é residencial, também cercado de comércios, de escolas sendo duas escolas municipais, duas creches, o colégio federal Pedro II, um abrigo para homens e mulheres, três universidades particulares e igrejas. A escolha do cenário se deu por ser um local com interfaces de ensino, assistência e pesquisa.

A análise de conteúdo utilizada foi de Bardin, onde ocorreu a condensação e o destaque das informações para análise, culminando nas interpretações inferenciais; com a intuição, da análise reflexiva e crítica, com a obtenção dos dados, foi efetuada uma análise do conteúdo através do levantamento das respostas obtidas pelo roteiro de perguntas e da transcrição integral das entrevistas. Os dados obtidos através se desdobraram em núcleos ou unidades que foram apresentados por categorias.

A clínica é uma unidade estratégica de saúde da família atuando na atenção primária, com uma população de cerca de 18.000 habitantes atendidos por 4 equipes durante a semana, nessas equipes estão três equipes de ESF com seis residentes. São 2 residentes por equipe sendo um R1 e um R2 e uma equipe. Ainda possui duas residências terapêuticas como responsáveis por uma equipe de Estratégia de Saúde da Família e um preceptor.

As entrevistas foram feitas com Enfermeiros Residentes e Preceptores enfermeiros que estão há mais de 6 meses na unidade de saúde. A pesquisa foi feita após contato com a gerente da ESF em dias agendados e local reservado para a entrevista. O período em que foi realizada a entrevista na clínica da família foi no mês de agosto de 2021.

Para participar desta pesquisa, os sujeitos foram esclarecidos que todos os dados obtidos seriam mantidos em sigilo e privacidade, sendo também orientados que em qualquer momento do processo poderiam desistir da pesquisa, caso se sentissem constrangidos com alguma pergunta, sem qualquer prejuízo. O Termo de Consentimento Livre e Esclarecido foi assinado por cada participante, atendendo ao que pede a Resolução 466/12. Para reservar a sua identidade, os participantes desta pesquisa foram identificados por nome de fruta.

Foram usados na pesquisa todos os protocolos e normas de segurança contra a COVID-19 (distanciamento social em todas as entrevistas, lavagem das mãos com água e sabão e higienizador à base de álcool no período entre as entrevistas, uso de máscaras N-95 e materiais individuais de pesquisa). Logo ao chegar na clínica foram verificadas as temperaturas dos pesquisadores para permitir que fosse realizado o estudo de campo. As questões de ética foram feitas em confidencialidade de dados dando garantia aos pesquisadores envolvidos neste estudo que não foram publicados dados ou informações que possibilitem sua identificação. $\mathrm{O}$ seu consentimento somente permitiu o uso de dados coletados referentes às atividades desenvolvidas de educação em saúde, cuidados de enfermagem e questões socioeducativas através do questionário para a pesquisa científica e fins educativos. 


\section{Resultados}

Quadro 1: Informações profissionais de cada enfermeiro(a), funções e tempo de atuação na clínica da Família na Zona Oeste do Rio de janeiro.

\begin{tabular}{lllllll}
\hline IDENTIDADE: & SEXO: & IDADE: & FUNÇÃO: & PERÍODO: & PÓS: \\
\hline MAÇÃ & FEM & 26 A 30 ANOS & RESIDENTE & 12 A 18 MESES & EM CURSO \\
\hline PERA & FEM & 20 A 25 ANOS & RESIDENTE & 12 A 18 MESES & EM CURSO \\
\hline MORANGO & FEM & 20 A 25 ANOS & RESIDENTE & 6 MESES & EM CURSO \\
\hline ABACAXI & FEM & 31 A 39 ANOS & PRECEPTORA & 12 A 18 MESES & SIM \\
\hline BANANA & FEM & 26 A 30 ANOS & RESIDENTE & 6 MESES & EM CURSO \\
\hline MELANCIA & FEM & 20 A 25 ANOS & RESIDENTE & 6 MESES & EM CURSO \\
\hline KIWI & MASC & 31 A 39 ANOS & RESIDENTE & 6 MESES & EM CURSO \\
\hline UVA & MASC & 26 A 30 ANOS & RESIDENTE & 6 MESES & EM CURSO \\
\hline MAMÃO & FEM & 20 A 30 ANOS & RESIDENTE & 6 MESES & EM CURSO \\
\hline
\end{tabular}

Fonte: Cidade; Gabriel (2021)

Essa Atenção Primaria tem preceptoria e residência em enfermagem há 2 anos, e participaram da pesquisa 7 enfermeiros do sexo feminino e 2 do sexo masculino, sendo 08 residentes e somente 1 preceptor, sendo 03 enfermeiros residentes do segundo ano de residência, (R2,) e a idade média de 25 anos, quanto a pós-graduação todos os residentes estão em curso e somente um entrevistado enfermeiro preceptor informou término de pós-graduação.

Quadro 2: Atendimento De Dor Precordial e Educação Permanente feito pelos Enfermeiros da Clínica da Família da Zona Oeste Rio de Janeiro.

\begin{tabular}{|l|c|c|}
\hline Atendimento dor precordial na ESF & N & \% \\
\hline Sim & $\mathbf{0 2}$ & $\mathbf{2 3}$ \\
\hline Não & 07 & \% \\
\hline $\begin{array}{l}\text { Encontros de educação permanente } \\
\text { na ESF }\end{array}$ & N & $\mathbf{2 3}$ \\
\hline Sim & $\mathbf{0 2}$ & 77 \\
\hline Não & 07 & $100 \%$ \\
\hline Total & 09 & \\
\hline
\end{tabular}

Fonte: Autores.

Observou-se no Quadro 2 Somente dois (23\%) dos entrevistados informaram, que a unidade de saúde atendeu usuários com dor precordial, inclusive neste período de pandemia no mês de julho de 2021, relataram que esses casos inclusive, foram de usuários sem uso de medicação anti-hipertensiva. Mas a unidade atende usuários com dor precordial, crise hipertensiva com regularidade, fora do período da pandemia Covid 19. 
Quanto à participação na educação permanente sobre prevenção do infarto, somente dois (23\%) participaram e foram dois residentes R2, durante este ano em julho de 2021. E somente um entrevistado (11\%) relatou que efetuou atendimento de usuário com dor precordial neste período de pandemia, sendo que o usuário foi atendido, mas em seguida encaminhado para o hospital, através do sistema de vaga zero.

Os grupos de promoção, roda de conversa e orientação aos usuários hipertensos e também de infarto agudo do miocárdio acontecem mensalmente na clínica da Família, mas devido esse período de pandemia em que os cuidados estão concentrados no atendimento a COVID 19 e a redução de funcionários na unidade de saúde em 2019 e 2020, 88\% informaram que não estão sendo feitos grupos de atendimento, somente tem ocorrido consulta de enfermagem e aconselhamento aos pacientes de risco.

\section{Categorização}

Separou-se as respostas às questões abertas por 4 categorias diversas, como demonstrado a seguir:

\section{Categoria 1: Ocorrência de grupos e/ou encontros com os usuários sobre prevenção de Infarto Agudo do Miocárdio:}

\section{Segundo relatos:}

Pêra, Maçã, Morango, Banana e Kiwi: "Grupos e encontros sobre autocuidado contra infarto e doenças cardiovasculares não têm ocorrido por conta da pandemia, mas os casos de pacientes de risco são feitos consulta de enfermagem de 15 em 15 dias ou mensalmente."

Abacaxi, Melancia, Mamão, Uva: "Aconselhamento nas consultas. sobre alimentação saudável e autocuidado".

A necessidade de intervenção da equipe de Saúde da Família, uma vez que a prevenção é a medida de ação em saúde necessária para reduzir os fatores de risco para as doenças coronarianas (Oliveira, 2015). Há necessidade de intervenção da equipe de Saúde da Família, pois as mudanças proativas para hábitos de vida mais saudáveis ainda são pequenas (Oliveira,2015).

\section{Categoria 2: Em tempo de covid o acompanhamento dos hipertensos na clínica da família de Realengo Zona Oeste} Segundo relatos como os atendimentos estão acontecendo:

Banana, Pera, Morango, Uva, Abacaxi, Kiwi e Melancia: "Demanda espontânea e consulta de enfermagem."

Segundo os relatos, sete enfermeiros (77\%) informaram que está ocorrendo a demanda espontânea e consulta de enfermagem. Observa-se que o acompanhamento dos usuários tem acontecido, através do fornecimento de receitas médicas pelos médicos da unidade. Caso haja relatos e o usuário estiver descompensado, deve ser acompanhado pelo profissional médico e enfermeiro, de acordo com a necessidade e já encaminhado para um hospital de pronto atendimento se necessário.

Maçã: "São realizados acompanhamento com os descompensados."

Os usuários estão sendo atendidos dentro das medidas preventivas de educação orientação à saúde pelos enfermeiros de acordo com o anexo 5 da portaria COFEN 384 de 5 de maio de 2021. Medidas de tomadas para evitar a contaminação da COVID 19 e medidas preventivas de educação e orientação à saúde no contato contínuo com usuários, principalmente os descompensados onde se dá um suporte. Os usuários descompensados estão sendo acompanhados pela equipe de saúde e apanham suas medicações na farmácia.

\section{Segundo relato abaixo de estar acontecendo renovação de receitas:}

Mamão: "Não está havendo acompanhamento, apenas em caso de renovação de receita."

Acredita-se que a "reorganização" da APS realizada pela atual gestão municipal tem impactos não só no acesso ao 
sistema de saúde e no acompanhamento de condições de saúde da população, como agrava ainda mais o cenário de pandemia que vivemos. Com a supressão de equipes e consequente redução na cobertura da ESF no município, os profissionais se viram sobrecarregados e sem condições de prestar a assistência devida à população.

Esses profissionais, que muitas vezes possuem fragilidades nas habilidades necessárias para o manejo clínico da Covid-19, são levados a atuar em ambientes desconhecidos e com limitações na oferta de equipamentos de proteção (Fehn,2020).

Todo esse contexto, que fragiliza ainda mais o profissional de saúde, causa desassistência e precariza o acompanhamento dos usuários da APS. Além disso, a atenção aos usuários com a Covid-19, que poderia ser mais próxima com o auxílio da APS, de modo a evitar o agravamento dos quadros a um ponto crítico, fica prejudicada pela situação que as equipes enfrentam. Considerando que as populações pobres são mais suscetíveis a ter doenças crônicas, isso os coloca em maior risco de mortalidade associada a Covid-19. A dificuldade do acesso, que já existia, acentua a vulnerabilidade desta população nesse momento de crise (Ahmed, 2020).

\section{Categoria 3: Orientações sobre o autocuidado que tem sido oferecido aos usuários hipertensos da clínica da família}

Pera: "Controle por usuário pelo ACS, exames de rotina, consultas de seis em seis meses para usuários controlados e 1 vez por mês do NASF."

A atuação NASF rotineira foi impactada significativamente, dadas a quantidade de atividades envolvendo trabalhos presenciais em grupo. Devido ao isolamento social, as equipes tiveram que suspender os grupos de atividades de educação e promoção da saúde, e reinventar o próprio trabalho de atenção à população. As equipes e a coordenação municipal NASF ajudaram as equipes ESF no planejamento das ações, na discussão de casos em andamento.

Maçã, Banana, Kiwi, Uva e Mamão: "Alimentação, prática não farmacológica, dieta hipossódica, atividade física e encaminhamento para o NASF. Possui nutricionista, terapeuta ocupacional."

Morango, Abacaxi, Melancia: “Apoio em exercícios físicos, alimentação e controle pressórico.”

Sobre a orientação aos pacientes hipertensos foi comunicado para praticarem o autocuidado na alimentação consumindo alimentos saudáveis; preferir alimentos fonte de potássio, bem lavados, evitar o consumo de álcool, café e cigarro; não usar nenhum medicamento sem antes consultar seu médico; a prática de atividades físicas dentro das suas limitações na sua residência e redução do colesterol e triglicerídeos.

O NASF não é uma estrutura desvinculada da atenção primária à saúde e, como esta, tem dentre seus pressupostos a territorialização; a educação permanente em saúde; integralidade; participação social; promoção da saúde e humanização. Ele procura ampliar, aperfeiçoar a atenção e a gestão da saúde na ESF, superando uma lógica fragmentada e privilegiando a construção de redes de atenção e cuidado, constituindo-se em apoio às equipes de Saúde da Família (Brasil, 2015).

\section{Categoria 4: A autonomia do Enfermeiro na Promoção e Prevenção de agravos de usuários}

Banana, Maçã e Pera: "Autonomia total, pedido de exames e atuação na orientação."

Abacaxi, Melancia, Uva, Kiwi e Morango: "Na atenção básica tem-se muita autonomia em exames, orientações, atendimento, encaminhamentos.

Alguns protocolos também definem os conceitos e os valores profissionais que devem embasar a prática, o funcionamento dos serviços de saúde e as funções ou atribuições de cada membro da equipe de enfermagem; descrevem as técnicas ou os procedimentos de enfermagem, assim como as metas assistenciais, quando existentes, o que inclui o número de consultas e visitas por categoria profissional (Raglione, 2016). 
Mamão: "Autonomia um pouco restrita por conta da renovação de receita médica, mas em conta sobre orientação, o enfermeiro tem muita autonomia."

Os enfermeiros reconhecem, em seu fazer, potencialidades, como: exercer a prática clínica por meio da consulta de enfermagem, criar vínculos com a população e estabelecer com a equipe relações interpessoais que propiciem um ambiente de trabalho produtivo, saudável e satisfatório (Ferreira,2018).

É importante acentuar que todos os profissionais de saúde podem fazer acolhimento ao escutar a queixa, os medos e as expectativas, identificar risco e a vulnerabilidade, e acolher também a avaliação do próprio usuário. Responsabilizar-se pela resposta ao usuário vai necessariamente colocar em ação uma rede multidisciplinar de compromisso com essa resolução.

\section{Discussão}

Foram feitas 09 entrevistas com enfermeiros que atuam em uma unidade de Estratégia Saúde da Família no bairro de Realengo sendo $77 \%$ do sexo feminino, alto número de enfermeiras por ser uma característica da profissão que se iniciou somente com mulheres segundo Florence Nightgalle sendo que no momento, a existência de enfermeiro sexo masculino esta bem expressivo, o profissional de enfermagem do sexo masculino não enfrenta mais tantos preconceitos e o número de enfermeiros homens tem aumentado constantemente no mercado.

Os profissionais são jovens na sua maioria de 20 a 25 anos $55 \%$ e são residentes de enfermagem cuja carga horária se justifica 60 horas para os residentes e 40 horas para os preceptores ou enfermeiros de equipe da Equipe da Estratégia Saúde da Família. Os residentes R1 são os que têm 6 meses de atuação e os demais residentes estão no seu segundo ano, que são 22,2\% e demais profissionais enfermeiros de equipe com mais de 1 ano de atuação. $33.3 \%$.

O residente é um profissional em formação e cabe ao preceptor fazer a interlocução entre os espaços de aprendizagem e ser um profissional de referência para o profissional em formação. É função do preceptor de ESF toda área administrativa e são responsáveis pela orientação dos residentes nas atividades relativas ao campo e ao núcleo, devendo promover a integração entre os diferentes profissionais em formação, destes com a equipe de saúde, com a população e com os demais serviços com que se relacionaram durante o desenvolvimento da Residência Multiprofissional (Autônomo, 2015).

A Resolução SMS nº 4783 de 19 de fevereiro de 2021 instituiu uma comissão permanente de Sistematização da Assistência de Enfermagem para Atenção Primária à Saúde com foco no atendimento de doenças hipertensas que foram prejudicadas na sua integralidade, longitudinalidade e na equidade do cuidado devido a presente epidemia que assola nosso país.

Obtivemos relatos de profissionais nos quais os doentes crônicos estavam tendo acesso a renovação de receita e recebendo as medicações prescritas e de uso contínuo na farmácia. E também houve relatos de utilização do telefone para retirada de dúvidas dos usuários.

Quanto às orientações sobre o autocuidado tem sido oferecido aos usuários hipertensos algumas práticas estavam sendo realizadas como: não-farmacológicas, a manutenção de orientação da dieta, as orientações sobre as atividades físicas na própria residência estavam sendo feitas pela equipe do NASF.

Ocorreu também a diminuição nos agendamentos de consultas, sendo assim, a procura que houve foi em relação à demanda espontânea, correlacionados à renovação de receitas e exames periódicos, por que no período da pesquisa estava ocorrendo a Pandemia da Covid 19. Segundo a Resolução Conjunta SEOP SMS nº 010 de 27 de maio de 2021 , que regulamenta a atuação em razão das medidas emergenciais de enfrentamento da Covid-19, de acordo com as necessidades que a clínica impõe. 
A educação permanente não foi realizada por impedimentos sanitários, mas, sempre que possível, os usuários que procuravam a unidade de saúde eram assistidos de acordo com a sua necessidade. porém a estratificação de risco, também atuou na vigilância epidemiológica de usuários que poderiam complicar o quadro clínico por conta de doenças coronarianas.

\section{Conclusão}

Observou-se a autonomia que o enfermeiro possui na clínica da família em relação ao acompanhamento e prescrição de acordo com os programas do ministério da saúde. Na pesquisa foi visto que durante todo o período, o atendimento de usuários crônicos, estavam sendo feitos por demanda espontânea através de renovação de receitas e teve uma redução de atendimento e atividades de educação permanente, por conta da contingência e restrições sanitárias na unidade de saúde, devido a COVID 19. Apenas os usuários de risco, motivados por dor precordial ou que estivessem descompensados, estavam sendo atendidos. Acontecia também algumas práticas não farmacológicas aos usuários em relação a alimentação e cuidados. A atividade de educação permanente acontece nesta clínica da família, e os enfermeiros são envolvidos nos programas de saúde e atividades afins de educação, mas neste período de Pandemia pelo acúmulo de atendimentos, adoecimento dos profissionais, ocorrência da imunização e exames para detectar COVID 19, não estava acontecendo atividades de educação permanente.

A reflexão tem por sua finalidade, trazer a importância vinculada a novas pesquisas e estudos com a temática Prevenção do infarto agudo do miocárdio feita pelos enfermeiros em uma clínica da família de Realengo, Rio de Janeiro. Tendo por seu papel principal, estratégias e medidas traçadas acerca da necessidade de um plano e ações de cuidados diferenciados, colocando em pauta a adequação e complexidade do paciente. Relevando também um bom conhecimento e as potenciais complicações no decorrer do tratamento, abordando da melhor forma com qualidade e agilidade, mantendo-se sempre como base o bem estar.

\section{Referências}

Andrade, J. P. D., Mattos, L. A. P., Carvalho, A. C., Machado, C. A., \& Oliveira, G. M. M. D. (2015). Programa nacional de qualificação de médicos na prevenção e atenção integral às doenças cardiovasculares. Arquivos Brasileiros de Cardiologia, 100(3), 203-211.

Básica, A. (2015). Saúde na escola. Ministério da Saúde.

Bolzan, E. P., \& Pompermaier, C. (2020). Cuidados de enfermagem ao paciente com Infarto Agudo do Miocárdio. Anuário Pesquisa e Extensão Unoesc Xanxerê, 5, e24115-e24115.

Brunori, E. H. F. R., Lopes, C. T., Cavalcante, A. M. R. Z., Santos, V. B., Lopes, J. D. L., \& Barros, A. L. B. L. D. (2015). Associação de fatores de risco cardiovasculares com as diferentes apresentações da síndrome coronariana aguda. Revista Latino-Americana de Enfermagem, $22,538-546$.

de Oliveira, L. B., \& de Araújo Püschel, V. A. (2015). Conhecimento sobre a doença e mudança de estilo de vida em pessoas pós-infarto. Revista Eletrônica de Enfermagem, 15(4), 1026-33.

dos Santos, L. C. A., Ribeiro, W. A., do Carmo Neves, K., Fassarella, B. P. A., Alves, A. L. N., de Castro, K., \& da Silva, D. H. S. (2021). Avaliação da dor neonatal na perspectiva do enfermeiro. Recisatec-revista científica saúde e tecnologia, 1(5), e1552-e1552.

Guimarães, D. B. O., Rodrigues, T. S., Oliveira, S. C. M., \& Avelino, F. V. S. D. (2018). Tempo porta eletrocardiograma em pacientes com dor torácica na emergência. Rev. enferm. UFPE on line, 1027-1036.

Mbilinyi, M., \& Atlas, T. B. (2021). Punctuated evolution in the learned songs of African sunbirds.

Meneses, L. S. L., Caxias, A. M., Franco, A. M., Dantas, A. S. F., de Oliveira, A. K. C., Leal, E. G., \& Miranda, Y. F. (2020). Assistência de enfermagem ao paciente com infarto agudo do miocárdio em uma urgência e emergência: relato de experiência. Brazilian Journal of Development, 6(9), 69116-69121.

Moraes, C. L. K., Tessmann, M., Botelho, R. V., Mendonça, V., Schmitt, A., \& Ficagna, F. T. (2020). Perfil e tempo porta-balão de pacientes com infarto agudo do miocárdio. Revista Inova Saúde, 10(2), 107-124.

Oliveira, L. A. M., \& DE, M. (2019). Cuidados de enfermagem ao paciente com infarto agudo do miocárdio: uma revisão integrativa. Brazilian Journal of sugery and clinical research, 28(3), 77-79.

Passinho, R. S., Sipolatti, W. G. R., Fioresi, M., \& Primo, C. C. (2018). Sinais, sintomas e complicações do infarto agudo do miocárdio. Rev. enferm. UFPE on line, 247-264.

Piegas, L. S., Timerman, A., Feitosa, G. S., Nicolau, J. C., Mattos, L. A. P., Andrade, M. D., ... \& Mathias, W. (2015). V Diretriz da Sociedade Brasileira de 
Research, Society and Development, v. 11, n. 3, e23711326484, 2022

(CC BY 4.0) | ISSN 2525-3409 | DOI: http://dx.doi.org/10.33448/rsd-v11i3.26484

Cardiologia sobre tratamento do infarto agudo do miocárdio com supradesnível do segmento ST. Arquivos brasileiros de cardiologia, 105,1 -121.

Prieto, N. G. (2021). Aspectos biopsicossociais e sexuais entre mulheres climatéricas em uma região do Sul do Brasil: um estudo quantitativo.

Raglione, D., Bezerra, G. A. M., Lopes, M. H., Nerger, M. L. B. R., Guimarães, T. C., \& Sartori, A. M. C. (2016). Avaliação da rede de frio para conservação de vacinas em unidades básicas de saúde das regiões Sul e Centro-Oeste do município de São Paulo em 2011-2012. Epidemiologia e Serviços de Saúde, 25, $65-74$.

Santos, B. D. S., Maier, S. R. D. O., Rodrigues, A. Z., Oliveira, W. D. S., \& Santos, T. D. S. (2017). Infarto agudo do miocárdio: abordagem com enfermeiros de uma unidade intensiva coronariana. Rev. enferm. UFPE on line, 5153-5155.

Santos, J. D., Meira, K. C., Camacho, A. R., Salvador, P. T. C. D. O., Guimarães, R. M., Pierin, Â. M. G., \& Freire, F. H. M. D. A. (2018). Mortalidade por infarto agudo do miocárdio no Brasil e suas regiões geográficas: análise do efeito da idade-período-coorte. Ciência \& saúde coletiva, 23, $1621-1634$.

Santos, W. da S., Gonçalves, N. J. de M. C., \& Viana, M. R. P. (2020). Nursing assistance to patients with acute myocardial infarction submitted to coronary angioplasty. Research, Society and Development, 9(7), e755974878. https://doi.org/10.33448/rsd-v9i7.4878

Souza, E. C. F. D., Vilar, R. L. A. D., Rocha, N. D. S. P. D., Uchoa, A. D. C., \& Rocha, P. D. M. (2015). Acesso e acolhimento na atenção básica: uma análise da percepção dos usuários e profissionais de saúde. Cadernos de Saúde Pública, 24, s100-s110.

Vargas, R. A. D., Riegel, F., Oliveira Junior, N. J. D., Siqueira, D. S., \& Crossetti, M. D. G. O. (2017). Qualidade de vida de pacientes pós-infarto do miocárdio: revisão integrativa da literatura. Revista de Enfermagem UFPE On Line. Recife. 11(7),. $2803-2809$. 